\title{
Semileptonic B Decays from CLEO
}

\author{
Karl M. Ecklund* \\ Newman Laboratory of Nuclear Studies \\ Cornell University, Ithaca, New York, USA \\ E-mail: 'kme@mail. Ins. cornell .edü
}

ABSTRACT: I report results in semileptonic decays of $B$ mesons from the CLEO collaboration, with a focus on recent results. Results for exclusive reconstruction of $B \rightarrow D^{*} \ell \nu, B \rightarrow D \ell \nu$ and $B \rightarrow \rho \ell \nu$ are given including the $q^{2}$ dependence of the form factors. These results are used to measure $\left|V_{c b}\right|$ and $\left|V_{u b}\right|$. Two preliminary analyses using inclusive techniques measure the lepton momentum spectrum and hadronic recoil mass spectrum in $B \rightarrow X_{c} \ell \nu$ decays, showing promise for future precision measurements of $\left|V_{c b}\right|$.

Study of semileptonic decays of $B$ mesons allows measurement of the CKM matrix elements

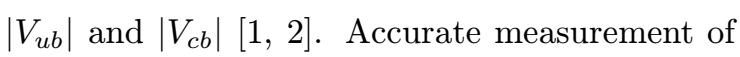
CKM matrix elements becomes increasingly important as we enter the era of the $B$-Factories and studies of $C P$ violation in $B$ meson decays.

In the Standard Model, $C P$ violation comes about through a non-zero phase in the CKM matrix, which describes quark mixing in weak decays. Decays of the $b$-quark in particular will be key to our understanding of $C P$ violation and flavour physics. In the well known unitarity triangle, $\left|V_{c b}\right|$ sets the overall scale for the length of the sides, and $\left|V_{u b}\right|$ determines the length of one side. Precise determinations of both will be needed to complement the measurement of the angles of the unitarity triangle in view at the $B$-Factories. The goal is to over-constrain the unitarity triangle and test the Standard Model.

In principle, CKM matrix elements can be studied in any weak decay mediated by the $W$ boson. Semileptonic decays (see figure $\left.\overline{1}_{1}^{1}\right)$ ) offer the advantage that the leptonic current is calculable and QCD complications only arise in the hadronic current. Unlike hadronic decays, there are no final state interactions and only one amplitude contributes to the decay. One still needs some understanding of the strong interaction. Heavy Quark Effective Theory and other

\footnotetext{
*Representing the CLEO Collaboration
}

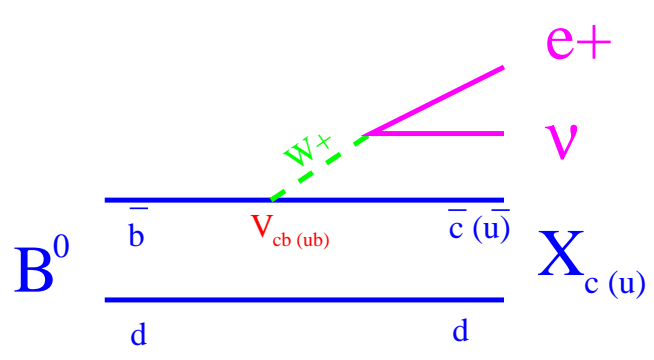

Figure 1: Diagram for semileptonic $B$ decay.

approaches to QCD calculations such as light cone sum rules and lattice QCD offer detailed and robust predictions for the QCD dynamics in heavy quark decay. These predictions allow measurement of $\left|V_{u b}\right|$ and $\left|V_{c b}\right|$ with reasonable precision. Data from experiment are beginning to test our understanding of heavy quark decay. As theory and experiment interact, an improved understanding will lead to more precise measurements.

In what follows, I present results in exclusive and inclusive semileptonic decays from the CLEO collaboration. We study the exclusive decays $B \rightarrow D^{(*)} \ell \nu$ to measure $\left|V_{c b}\right|$ and the form factors which describe the role of the strong interaction in the decay. In $B \rightarrow \pi(\rho) \ell \nu$ we study the form factor for $B$ transitions to light mesons and measure $\left|V_{u b}\right|$. Inclusive measurements of 
$B \rightarrow X_{c} \ell \nu$ offer comparable precision on $\left|V_{c b}\right|$ with new techniques that constrain phenomenological parameters using decay spectra.

\section{CLEO Experiment}

CLEO is a $4 \pi$ solenoidal detector located at the interaction region of the Cornell Electron Storage Ring (CESR). CESR is a symmetric $e^{+} e^{-}$ collider operating on the $\Upsilon(4 S)$ resonance at a center of mass energy of $10.58 \mathrm{GeV}$, just above the threshold for $B \bar{B}$ production. $\Upsilon(4 S)$ decays are essentially $100 \% B_{d}^{0} \bar{B}_{d}^{0}$ and $B^{+} B^{-}$pairs. At threshold the $B$ 's are produced nearly at rest: $p_{B} \approx 300 \mathrm{MeV} / c$. In addition to $\Upsilon(4 S)$ production with a cross section of $1.0 \mathrm{nb}$, there is continuum production (3.0 nb) of $e^{+} e^{-} \rightarrow$ hadrons. CLEO also collects data $60 \mathrm{MeV}$ below the $\Upsilon(4 S)$ for use in subtraction of this continuum from onresonance data.

The central region of the CLEO detector consists of three concentric cylindrical drift chambers, a scintillator time-of-flight system and a CsI calorimeter all inside a superconducting coil and $1.5 \mathrm{~T}$ magnetic field. Endcap time-of-flight and CsI calorimeters provide forward and backward coverage for a total of $95 \%$ of the solid angle. The drift chambers provide excellent tracking and momentum resolution, and the calorimeter has excellent photon and $\pi^{0}$ identification. In the flux return for the superconducting solenoid, proportional tube counters provide muon identification at depths of 3,5 and 7 interaction lengths. The CLEO detector is described in detail elsewhere [3]

\section{2. $B \rightarrow D^{*} \ell \nu$}

As an example of how one can extract $\left|V_{c b}\right|$ from an exclusive semileptonic $B$ decay, consider $B \rightarrow$ $D^{*} \ell \nu$. The differential decay rate is given by equation 12.

$$
\frac{d \Gamma}{d w}=\frac{G_{F}^{2}}{48 \pi^{3}}\left|V_{c b}\right|^{2} \mathcal{F}_{D^{*}}^{2}(w) \mathcal{G}(w)
$$

Here $w=\left(M_{B}^{2}+M_{D *}^{2}-q^{2}\right) /\left(2 M_{B} M_{D *}\right)$ is the inner product of the heavy quark four velocities, which is linearly related to $q^{2}$, the invariant mass of the $W$. The function

$$
\begin{aligned}
\mathcal{G}(w) & =M_{D^{*}}^{3}\left(M_{B}-M_{D^{*}}\right)^{2} \sqrt{w^{2}-1}(w+1)^{2} \\
& \times\left[1+\frac{4 w}{w+1} \frac{1-2 w r+r^{2}}{(1-r)^{2}}\right]
\end{aligned}
$$

with $r=M_{D^{*}} / M_{B}$, is a function of $w$ and the relevant masses. The function $\mathcal{F}_{D^{*}}(w)$ is the form factor for the $B$ to $D^{*}$ transition.

In the limit of very heavy quarks $\left(M_{b, c} \rightarrow\right.$ $\infty), \mathcal{F}(w)$ becomes the universal Isgur-Wise function, and $\mathcal{F}(1)=1$. That is, the form factor is known with absolute normalization at the point of zero recoil, or $w=1$. For finite heavy quark masses the corrections may be computed in the framework of Heavy Quark Effective Theory (HQET). The QCD corrections are computed in a perturbation theory using $\Lambda_{\mathrm{QCD}} / M_{b}$ and $\alpha_{s}$ as expansion parameters. For $B \rightarrow D^{*} \ell \nu$ the first order correction vanishes exactly (known as Luke's

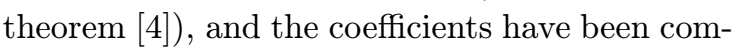
puted to order $\alpha_{s}^{2}$ and $1 / M_{b}^{2}\left[\begin{array}{ll}1 \\ 1\end{array}\right.$

$$
\mathcal{F}_{D^{*}}(w=1)=0.91 \pm 0.03 .
$$

The value above is in agreement with the assessment of The BaBar Physics Book authors [9.1], but Bigi has recently given a central value of 0.89 , with substantially larger uncertainty [100i]"

In an experiment one measures the decay rate as a function of $w$ and extrapolates to $w=$ 1. At this kinematic point, the $D^{*}$ has no momentum in the rest frame of the $B$ meson. At CLEO, where the $B$ 's are produced near threshold, the momentum of the resulting slow pion from $D^{*+} \rightarrow D^{0} \pi^{+}$is very small. The efficiency for reconstructing the slow pion approaches zero as one approaches the zero-recoil point, making the extrapolation more difficult. This experimental difficulty is avoided for $B^{-} \rightarrow D^{* 0} \ell \bar{\nu}$, where a slow $\pi^{0}$ may be reconstructed even at very small momenta.

As the kinematically allowed range of $w$ is small $(w \in[1.0,1.5])$, the form factor is approximated as a Taylor expansion about $w=1$.

$$
\mathcal{F}(w)=\mathcal{F}(1)\left(1+(w-1) \rho^{2}+c(w-1)^{2}\right)
$$

CLEO has measured the $B \rightarrow D^{*} \ell \nu$ decay rate as a function of $w$ as shown in figure The figure also shows $\mathcal{F}(w)\left|V_{c b}\right|$ versus $w$ (the 

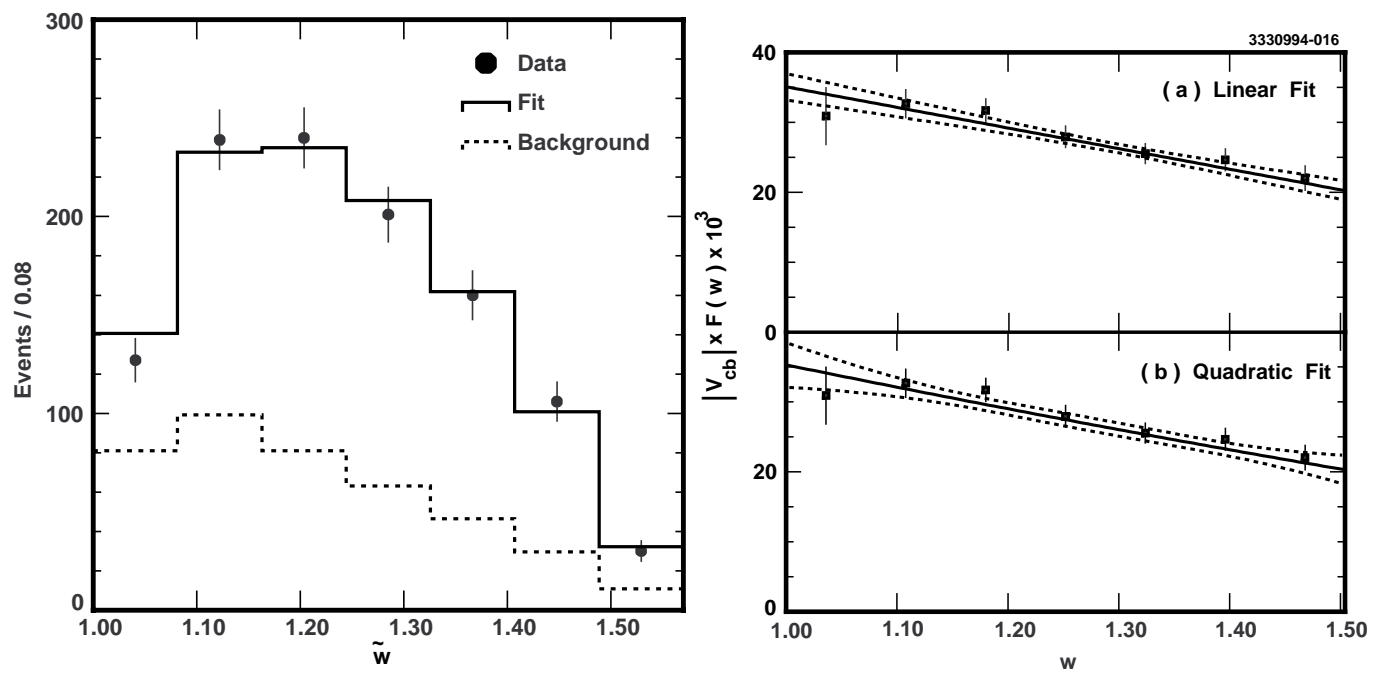

Figure 2: The left side shows the differential yield $d N / d w$ for $B^{-} \rightarrow D^{* 0} \ell^{-} \bar{\nu}$ and $\bar{B}^{0} \rightarrow D^{*+} \ell^{-} \bar{\nu}$. The fit shown is to a linear form factor. The background is mostly due to combinatoric $D^{*}$ candidates. On the right $\mathcal{F}(w)\left|V_{c b}\right|$ is plotted vs $w$. Data points are overlayed with fit results (solid line) for linear (a) and quadratic (b) form factors. The dashed lines show the contours for $1 \sigma$ variations of the fit parameters.

kinematic factors have been divided out) showing that experiment currently does not differentiate between a linear and quadratic dependence on $w$ for the form factor. Taking the linear fit, one may read off the value for $\mathcal{F}(1)\left|V_{c b}\right|$ at the intercept,

$\mathcal{F}_{D^{*}}(1)\left|V_{c b}\right|=0.0350 \pm 0.0019 \pm 0.0018 \pm 0.0003$.

The uncertainties are statistical, the systematic (dominated by slow pion efficiency) and due to the $B$ lifetime. I have updated the result using the PDG98 $B$ lifetimes [i12n. Using $\mathcal{F}(1)=0.91 \pm$ 0.03 , this gives

$$
\left|V_{c b}\right|=0.0385 \pm 0.0029 \pm 0.0013 .
$$

This result is based on 1.6 million $B \bar{B}$ pairs. CLEO currently has nearly 10 million $B \bar{B}$ events, so substantial improvement in this measurement may be expected. LEP experiments also use this technique to measure $\left|V_{c b}\right|\left[\begin{array}{l}1 \\ 1\end{array} \overline{3}\right.$ ] using a smaller number of $B$ decays, with somewhat different experimental systematic uncertainties. For CLEO, the leading systematic comes from modelling of the slow pion efficiency. At LEP, contributions from semileptonic decay to higher excited charm mesons $\left(B \rightarrow D^{* *} \ell \nu\right)$, which are poorly known, lead to non-negligible systematic uncertainties.

\section{3. $B \rightarrow D \ell \nu$}

The decay $B \rightarrow D \ell \nu$ can be analyzed in the same way as the $D^{*} \ell \nu$ decay described above, although the precision with which $\left|V_{c b}\right|$ can be determined is not as good because of a smaller branching fraction, larger backgrounds and an additional kinematic suppression factor of $w^{2}-1$. (Compare equations $\overline{2} .2$ and $3 . \overline{2}$. ) Nonetheless it provides complementary information and provides a test of HQET predictions for relationships between the form factors for semileptonic decays of pseudoscalar $(B)$ to pseudoscalar $(D)$ and pseudoscalar to vector $\left(D^{*}\right)$. In HQET to first order there is a common form factor, the Isgur-Wise function $\xi(w)$.

CLEO has two recent analyses of $B \rightarrow D \ell \nu$. The first [1] nique to completely reconstruct the decay chain.

In the second analysis [15in], we identify events containing a $D^{+}$or $D^{0}$ (and charge conjugates) and an electron or muon $(\ell)$. The $D-\ell$ combinations give a sample including $B \rightarrow D \ell \nu$, $B \rightarrow D^{*} \ell \nu, B \rightarrow D^{* *} \ell \nu$ and $B \rightarrow D^{(*)} \pi \ell \nu$. We separate $B \rightarrow D \ell \nu$ from the other semileptonic modes using the energy and momentum of the particle(s) recoiling against the $D-\ell$ pair. The yield of $D \ell \nu$ events in bins of $q^{2}$, the invari- 


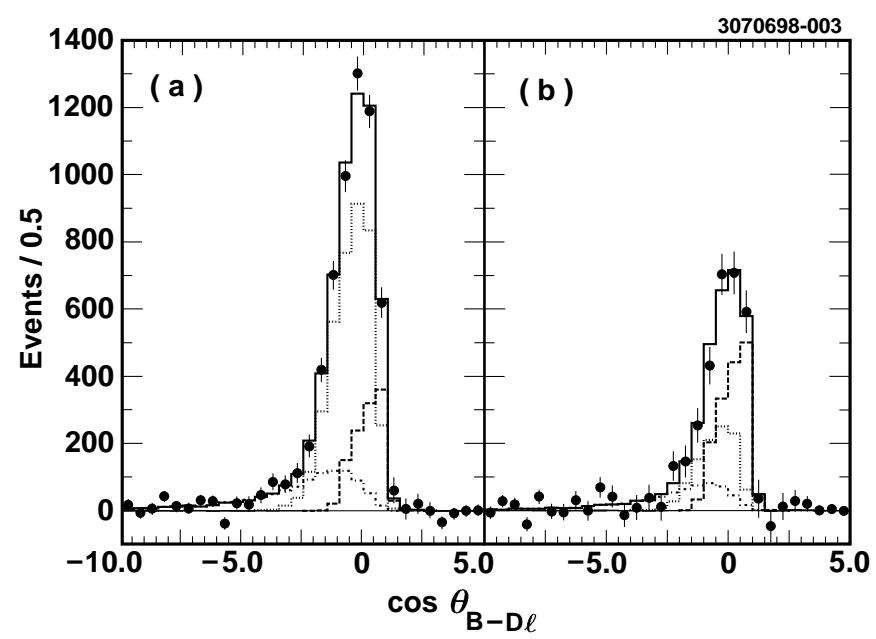

Figure 3: The $\cos \theta_{B-D \ell}$ distribution for (a) $D^{0} X \ell \nu$ and (b) $D^{+} X \ell \nu$ candidates. The data (solid circles) are overlayed with simulated $B \rightarrow D \ell \nu$ decays (dashed histogram), $B \rightarrow D^{*} \ell \nu$ decays (dotted histogram), $B \rightarrow D^{* *} \ell \nu+D^{(*)} \pi \ell \nu$ decays (dash-dotted histogram), and their total (solid histogram).

ant mass of the virtual $W$, gives information on the partial width and form factors in the decay $B \rightarrow D \ell \nu$.

We reconstruct $D$ candidates in the modes $D^{0} \rightarrow K^{-} \pi^{+}$or $D^{+} \rightarrow K^{-} \pi^{+} \pi^{+}$, separating $K$ and $\pi$ tracks by using time-of-flight and drift chamber $d E / d x$ measurements. To suppress $D$ 's from the continuum, we require the $D$ candidates to have momentum $p_{D}<2.5 \mathrm{GeV} / c$. We select electron candidates of momentum $0.8<p_{\ell}<2.4$ $\mathrm{GeV} / c$ using the CsI calorimeter. Muon candidates must have associated hits in the muon counters, penetrating at least 5 interaction lengths of material, which increases the lower momentum cut for muons to $1.4 \mathrm{GeV} / c$. For $90 \%$ of signal $D \ell \nu$ events, the lepton and $D$ lie in opposite hemispheres; we require this of all $D-\ell$ pairs.

For each $D-\ell$ pair we compute $\cos \theta_{B-D \ell}$, the angle between the $D \ell$ momentum and the $B$ momentum assuming that the only missing particle is a massless neutrino.

$$
\cos \theta_{B-D \ell}=\frac{2 E_{B} E_{D \ell}-M_{B}^{2}-M_{D \ell}^{2}}{2\left|\mathbf{p}_{B}\right|\left|\mathbf{p}_{D \ell}\right|}
$$

For $B \rightarrow D \ell \nu$ decays $\cos \theta_{B-D \ell}$ lies between 1 and -1 . When final state particles other than the neutrino are missing, it is shifted towards negative values. Thus we may use this quantity to distinguish $D \ell \nu$ from $D X \ell \nu$. Before doing so other backgrounds must be subtracted.
Background sources yielding a $D-\ell$ pair may arise from (1) random $K \pi(\pi)$ combinations (fake $D)$, (2) a $D$ paired with a lepton from the other $B$ decay (uncorrelated), (3) a $D$ paired with a lepton that is a granddaughter of the same $B$ (correlated), (4) misidentification of a hadron as a lepton, or (5) $e^{+} e^{-} \rightarrow q \bar{q}$ events. We remove backgrounds from fake $D$ candidates by using events in the $D$ mass sidebands. The uncorrelated background contribution is estimated from our data by flipping the direction of leptons in the same hemisphere as the $D$ candidate. The small amount of correlated background (e.g. from $B \rightarrow$ $\left.D^{(*)} \tau \nu, \tau \rightarrow \ell \nu \bar{\nu}\right)$ is removed using Monte Carlo (MC) simulation. Fake leptons and continuum events are subtracted using measured fake rates and off-resonance data.

In figure ' $3 \overline{-1}$ ' the resulting $\cos \theta_{B-D \ell}$ distributions are shown along with a fit to the data. We model the distributions in the fit using MC simulation and various models for $b \rightarrow c$ semileptonic decay: for $B \rightarrow D \ell \nu$ we use ISGW2 [1] $\overline{6}^{1}, \overline{1} \overline{1} \overline{7}_{1}$; for $B \rightarrow D^{*} \ell \nu$ we use CLEO form factors [1 $\left.111,1,18_{1}^{1}\right]$; for $B \rightarrow D^{* *} \ell \nu$ we use ISGW2; and for non-resonant $B \rightarrow D^{(*)} \pi \ell \nu$ we use the results of Goity and Roberts [i1 $\bar{y}_{1}^{\prime}$. To extract form factor results we perform the fit to $\cos \theta_{B-D \ell}$ in ten bins of the HQET variable $w=\left(M_{B}^{2}+M_{D}^{2}-q^{2}\right) /\left(2 M_{B} M_{D}\right)$, where $q^{2}$ is the invariant mass of the $D-\ell$ pair. The $D \ell \nu$ yield in each $w$ bin is shown in figure 


\begin{tabular}{lccc} 
Form Factor & $\rho_{D}^{2}$ & $c_{D}$ & $\mathcal{F}_{D}(1)\left|V_{c b}\right| 10^{2}$ \\
\hline Linear & $0.76 \pm 0.16 \pm 0.09$ & - & $4.05 \pm 0.45 \pm 0.32$ \\
Free curvature & $0.77_{-2.83}^{+1.18} \pm 0.09$ & $0.01_{-3.96}^{+1.70} \pm 0.001$ & $4.05_{-1.63}^{+1.51} \pm 0.32$ \\
Boyd & $1.30 \pm 0.27 \pm 0.16$ & $1.21 \pm 0.31 \pm 0.15$ & $4.48 \pm 0.61 \pm 0.37$ \\
Caprini & $1.27 \pm 0.25 \pm 0.15$ & $1.18 \pm 0.26 \pm 0.14$ & $4.44 \pm 0.58 \pm 0.36$
\end{tabular}

Table 1: Summary of $B \rightarrow D \ell \nu$ form factor fits.

We fit the differential decay rate

$\frac{d \Gamma}{d w}=\frac{G_{F}^{2}\left|V_{c b}\right|^{2}}{48 \pi^{3}}\left(M_{B}+M_{D}\right)^{2} M_{D}^{3}\left(w^{2}-1\right)^{3 / 2} \mathcal{F}_{D}^{2}(w)$

assuming different form factors $\mathcal{F}_{D}(w)$. The fit accounts for detector acceptance and smearing in the reconstruction of $w$ due to motion of the $B$ and detector resolution $\left(\sigma_{w}=0.026\right)$. The

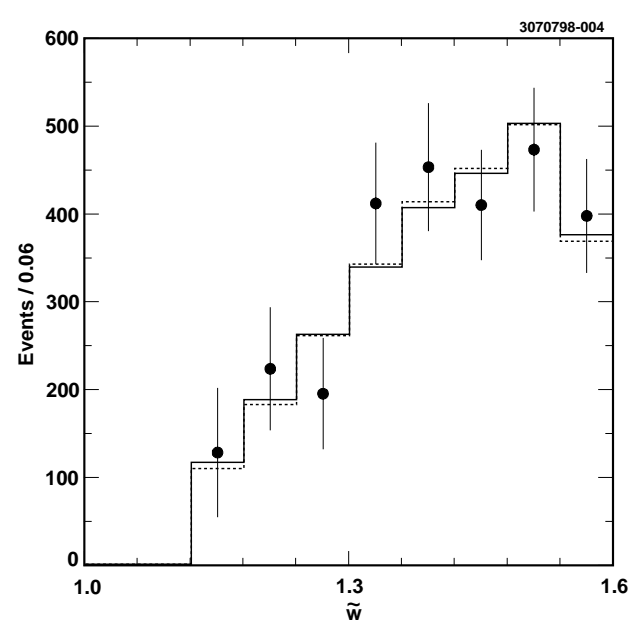

Figure 4: The sum of $B^{-} \rightarrow D^{0} \ell \bar{\nu}$ and $\bar{B}^{0} \rightarrow D^{+} \ell \bar{\nu}$ yields as a function of $\tilde{w}$, for the data (solid circles) and using the best fit linear form factor (dashed histogram) or dispersion relation inspired form factor of Boyd et al. (solid histogram).

results of the fit are given in table $1 \overline{1}$. We first parameterize the form factor as a Taylor expansion about $w=1$ :

$$
\mathcal{F}_{D}(w)=\mathcal{F}_{D}(1)\left(1-\rho_{D}^{2}(w-1)+c_{D}(w-1)^{2}\right) .
$$

We first fit using only a linear term $\left(c_{D}=0\right)$ and then include the curvature term. We also parameterize the form factor using the result of Boyd et al. [200], which uses dispersion relation to constrain terms of higher order in $(w-1)$. Similar results are obtained using the parameterization

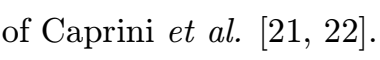

We obtain the total decay rate for $B \rightarrow D \ell \nu$ by integrating $d \Gamma / d w$ over $w$ using best fit values to Boyd et al.'s parameterization of the the form factor. We find $\Gamma(B \rightarrow D \ell \nu)=(14.1 \pm 1.0 \pm 1.2)$ $\mathrm{ns}^{-1}$, where we have combined $B^{-} \rightarrow D^{0} \ell^{-} \bar{\nu}$ and $\bar{B}^{0} \rightarrow D^{+} \ell-\bar{\nu}$ samples by assuming that $B^{0} \bar{B}^{0}$ and $B^{+} B^{-}$saturate the decays of the $\Upsilon(4 S)$. Using measured $B$ lifetimes this implies the branching fractions, $\mathcal{B}\left(B^{-} \rightarrow D^{0} \ell^{-} \bar{\nu}\right)=(2.32 \pm 0.17 \pm$ $0.20) \%$ and $\mathcal{B}\left(\bar{B}^{0} \rightarrow D^{+} \ell^{-} \bar{\nu}\right)=(2.20 \pm 0.16 \pm$ $0.19) \%$, where the errors are statistical and systematic respectively. Since we derive the branching fractions from the decay width, the errors are completely correlated. We combine these results with the previous CLEO measurement [1 14] taking into account all correlations:

$$
\begin{aligned}
& \Gamma(B \rightarrow D \ell \bar{\nu})=(13.4 \pm 0.8 \pm 1.2) \mathrm{ns}^{-1} \\
& \mathcal{B}\left(B^{-} \rightarrow D^{0} \ell^{-} \bar{\nu}\right)=(2.21 \pm 0.13 \pm 0.19) \% \\
& \mathcal{B}\left(\bar{B}^{0} \rightarrow D^{+} \ell^{-} \bar{\nu}\right)=(2.09 \pm 0.13 \pm 0.18) \% \\
& \mathcal{F}_{D}(1)\left|V_{c b}\right|=(4.16 \pm 0.47 \pm 0.37) \times 10^{-2}
\end{aligned}
$$

Theoretical expectations for $\mathcal{F}_{D}(1)$ range from $0.98 \pm 0.07[\overline{2} \overline{1}]$ to $1.04\left[\overline{1} \overline{6}_{\mathrm{n}}^{\prime} \overline{1} \overline{1} \overline{1}\right]$. A recent lattice calculation finds $1.058_{-0.017}^{+0.020}$ [233] $1.05 \pm 0.03$ we find

$$
\left|V_{c b}\right|=0.040 \pm 0.004 \pm 0.004 \pm 0.001
$$

where the errors are statistical, systematic and due to theoretical uncertainty in $\mathcal{F}_{D}(1)$. This value of $\left|V_{c b}\right|$ is consistent with that obtained in studies of the decay $B \rightarrow D^{*} \ell \nu$. If we use, instead, the best fit parameters to a linear form factor, the value of $\left|V_{c b}\right|$ decreases by $10 \%$. This shows the importance of the higher order terms in the form factor when extrapolating to $w=1$.

The form factor for $D \ell \nu$ may also be compared with $D^{*} \ell \nu$. In HQET at lowest order there is a common form factor. Corrections at higher 


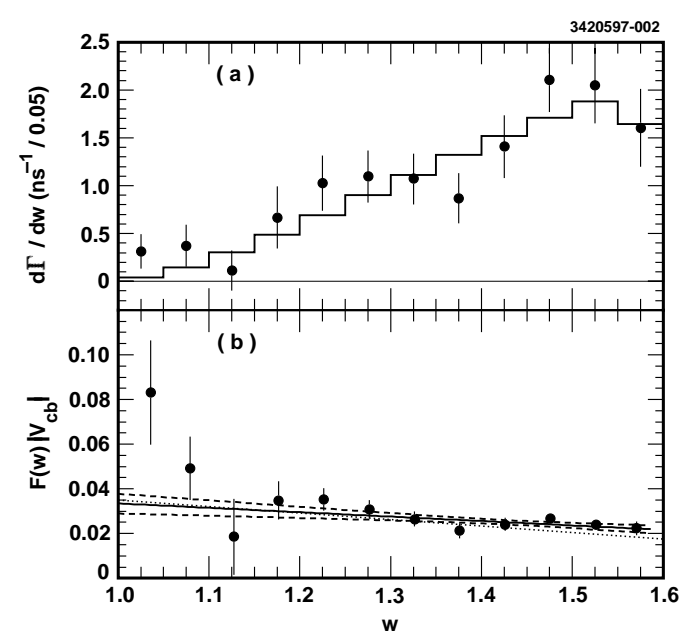

Figure 5: Overlay of $\mathcal{F}_{D^{(*)}}(w)\left|V_{c b}\right|$ where the points are $B \rightarrow D \ell \nu$ data [1] the solid line is the best fit with statistical errors shown by the dashed lines, and the dotted line shows the best fit from $B \rightarrow$ $D^{*} \ell \nu$ [i] 1 in.

order have also been calculated. Figure $\mathcal{F}(w)\left|V_{c b}\right|$ vs $w$ for both $D \ell \nu$ and $D^{*} \ell \nu$. With the statistics available the agreement is excellent as predicted by HQET.

\section{4. $B \rightarrow \pi \ell \nu$ and $B \rightarrow \rho \ell \nu$}

CLEO has also measured $b \rightarrow u \ell \nu$ decays which are sensitive to $\left|V_{u b}\right|$. Experimentally such measurements are difficult due to large backgrounds from the Cabibbo favored $b \rightarrow c \ell \nu$ decays.

\section{$4.11996 B \rightarrow \pi \ell \nu$ and $B \rightarrow \rho \ell \nu$ Analysis}

In 1996 CLEO observed the exclusive decay modes $B \rightarrow \pi \ell \nu$ and $B \rightarrow \rho(\omega) \ell \nu$ using the method of neutrino reconstruction [24]. Using the hermeticity of the CLEO detector, the neutrino momentum and energy are estimated to be the missing momentum and energy in the event.

$$
\begin{aligned}
& \mathbf{p}_{\nu}=-\sum_{i} \mathbf{p}_{i} \\
& E_{\nu}=2 E_{\text {beam }}-\sum_{i} E_{i}
\end{aligned}
$$

To ensure a good measurement of the missing energy and momentum, we require the net charge of the event to vanish and carefully identify showers in the crystal calorimeter to avoid double counting of interacting charged particles. To remove events with more than one neutrino, we require that there be only one charged lepton identified in the event. The neutrino mass inferred from the missing energy and momentum must be consistent with zero. The resolution on the missing energy (momentum) is $260 \mathrm{MeV}(110 \mathrm{MeV} / c)$.

Electrons are identified using the CSI calorimeter, and muons are identified by hits in the muon counters at depths of 5 and 7 interaction lengths. Backgrounds from $b \rightarrow c \ell \nu$ and $b \rightarrow c \rightarrow s \ell \nu$ are significantly reduced by requiring $p_{\ell}>1.5(2.0)$ $\mathrm{GeV} / c$ for the $\pi(\rho)$ mode. The $\rho^{ \pm}$and $\omega$ candidates are identified in the $\pi \pi$ and $\pi^{+} \pi^{-} \pi^{0}$ decay modes respectively.

The reconstructed neutrino 4 -vector $\left(\left|\mathbf{p}_{\nu}\right|, \mathbf{p}_{\nu}\right)$ is combined with a lepton and $\pi$ or $\rho$ candidate to form $B$ candidates, which will have the $B$ meson mass and beam energy for signal events. The signal is extracted by fitting the variables $M_{B}$ and $\Delta E$.

$$
\begin{aligned}
& \Delta E=E_{\pi(\rho)}+E_{\ell}+\left|\mathbf{p}_{\nu}\right|-E_{\text {beam }} \\
& M_{B}=\sqrt{E_{\text {beam }}^{2}-\left|\mathbf{p}_{\pi(\rho)}+\mathbf{p}_{\ell}+\mathbf{p}_{\nu}\right|^{2}}
\end{aligned}
$$

For $\rho \ell \nu$ we fit $M(\pi \pi)$ as well. The fit determines components for signal and background from $b \rightarrow$ $c$. Isospin and quark model relations are used to fix the relative rate of $B^{0}$ and $B^{+}$decays. We take $\Gamma\left(B^{0} \rightarrow \pi^{-} \ell^{+} \nu\right)=2 \Gamma\left(B^{+} \rightarrow \pi^{0} \ell^{+} \nu\right)$ and $\Gamma\left(B^{0} \rightarrow \rho^{-} \ell^{+} \nu\right)=2 \Gamma\left(B^{+} \rightarrow \rho^{0} \ell^{+} \nu\right) \approx$ $2 \Gamma\left(B^{+} \rightarrow \omega \ell^{+} \nu\right)$, leaving two independent yields $N_{\pi}$ and $N_{\rho}$. Cross-feed among the different modes and from $B \rightarrow X_{u} \ell \nu$ (higher resonances) is included in the fit. The fit result is shown in figure $\overline{6}$

We see clear signals in $B \rightarrow \pi \ell \nu$ and $B \rightarrow$ $\rho \ell \nu$. The yield in $B \rightarrow \omega \ell \nu$ is consistent both with the expectations from $\rho \ell \nu$ and with background. The branching fractions for the observed signals are determined using efficiencies from a full detector $\mathrm{MC}$ of the signals for five form fac-

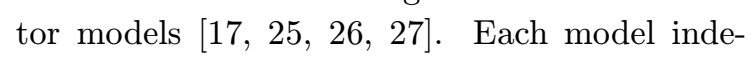
pendently predicts the $\pi / \rho$ ratio. We compare this to the data, and for four of the five models, the ratio is consistent. The remaining model is excluded, and we average the results from others.

$$
\begin{aligned}
& \mathcal{B}\left(B^{0} \rightarrow \rho^{-} \ell^{+} \nu\right)=\left(2.5 \pm 0.4_{-0.7}^{+0.5} \pm 0.5\right) \times 10^{-4} \\
& \mathcal{B}\left(B^{0} \rightarrow \pi^{-} \ell^{+} \nu\right)=(1.8 \pm 0.4 \pm 0.3 \pm 0.2) \times 10^{-4}
\end{aligned}
$$



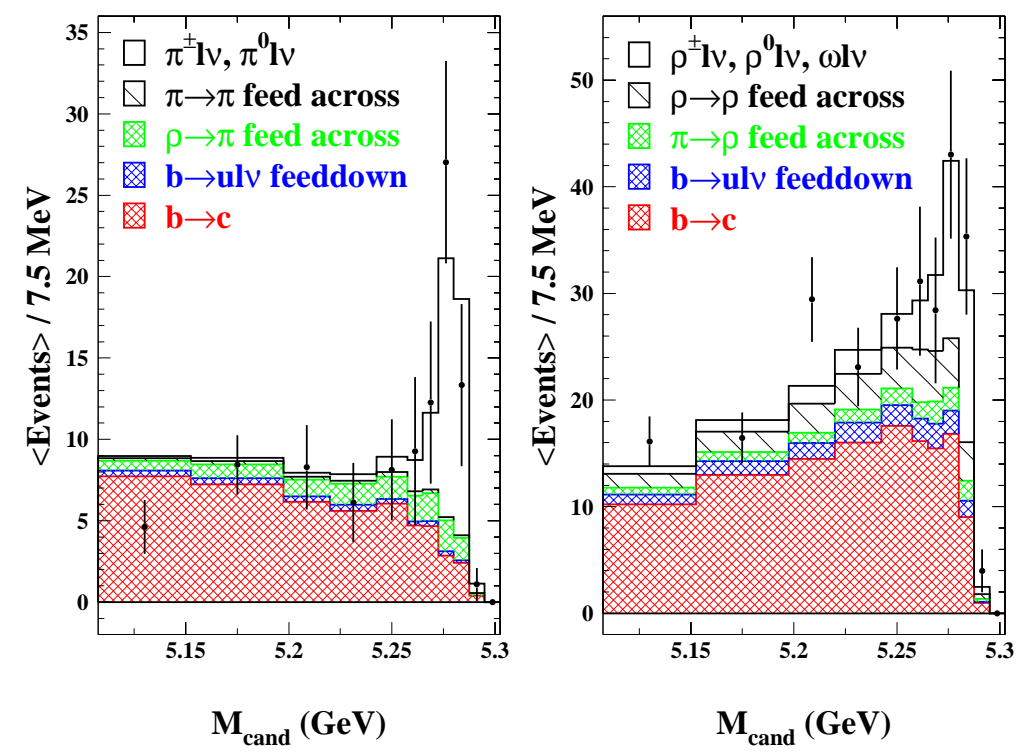

Figure 6: $M_{B}$ distributions from 1996 analysis showing $\pi \ell \nu$ (left) and $\rho \ell \nu$ (right).

$$
\left|V_{u b}\right|=\left(3.3 \pm 0.2_{-0.4}^{+0.3} \pm 0.7\right) \times 10^{-3}
$$

The uncertainties on the measurements are statistical, systematic and due to model dependence. The model dependence is estimated from the spread of the results using different models. This uncertainty $(\sim 20 \%)$ currently limits the accuracy of $\left|V_{u b}\right|$ measurements using exclusive $b \rightarrow u \ell \nu$ decays. However, with more $B$ decays, one can begin to differentiate between the different models. The aim of a second CLEO analysis is to increase the efficiency and begin to do just that by measuring the $q^{2}$ dependence.

\section{$4.21999 B \rightarrow \rho \ell \nu$ Analysis}

In this second analysis [28'], we analyze the decay $B \rightarrow \rho \ell \nu$ using high momentum leptons paired with $\pi, \rho$ and $\omega$ candidates. In the high momentum region we are able to measure the $q^{2}$ distribution of $B \rightarrow \rho \ell \nu$ events.

We select events with leptons of energy $E_{\ell}>$ $1.7 \mathrm{GeV} / c$ accompanied by a hadronic system consistent with a $\rho\left(\pi^{+} \pi^{-}\right.$or $\left.\pi^{ \pm} \pi^{0}\right), \omega\left(\pi^{+} \pi^{-} \pi^{0}\right)$ or $\pi\left(\pi^{ \pm}\right.$or $\left.\pi^{0}\right)$. To reduce background from $b \rightarrow$ $c \ell \nu$ decays we divide the sample into three lepton energy bins: HILEP $(2.3-2.7 \mathrm{GeV} / c)$, LOLEP $(2.0-2.3 \mathrm{GeV} / c)$ and LOLOLEP $(1.7-2.0 \mathrm{GeV} / c)$. Leptons in the HILEP bin have energy above the kinematic endpoint for $b \rightarrow c \ell \nu$ decays. The LOLEP bin contains mostly $b \rightarrow c \ell \nu$ events but still has some sensitivity to $b \rightarrow u \ell \nu$. The lowest energy bin provides a normalization of the $b \rightarrow c \ell \nu$ background.

The dominant source of background in the highest energy bin comes from continuum production of hadrons: $e^{+} e^{-} \rightarrow q \bar{q}, q=u, d, s, c$. Since the decays of $B$ mesons at rest are more spherical than jet-like $q \bar{q}$ events, we suppress this background using event shape variables. We obtain additional suppression by requiring $\cos \theta_{B-\rho \ell}$ to be physical. (See equation $3.1 i$ )

For each $\rho \ell \nu$ candidate, we compute $\Delta E=$ $E_{\rho}+E_{\ell}+\left|\mathbf{p}_{\text {miss }}\right|-E_{\text {beam }}$, where $\mathbf{p}_{\text {miss }}$ is the net missing momentum in the event. For signal events, $\Delta E$ should peak near zero since $\mathbf{p}_{\text {miss }}$ gives a measure of the neutrino energy and momentum. Because we rely on the hermeticity of the detector for this measurement, we require the missing momentum not to point down the beam pipe. We also require $\mathbf{p}_{\text {miss }}$ to be within $35^{\circ}$ of the neutrino direction inferred from the $\rho+\ell$ candidate; the later is known up to an azimuthal ambiguity about the $B$ momentum direction.

To measure the $\rho \ell \nu$ branching fraction, we perform a simultaneous maximum likelihood fit for all five modes in all three lepton energy bins. We fit in two variables, $\Delta E$ and $m(\pi \pi(\pi))$, for the $\rho$ and $\omega$ modes; for the $\pi$ modes, we fit only to $\Delta E$. The fit contains contributions from the physics processes $B \rightarrow \rho(\omega) \ell \nu, B \rightarrow \pi \ell \nu$, 

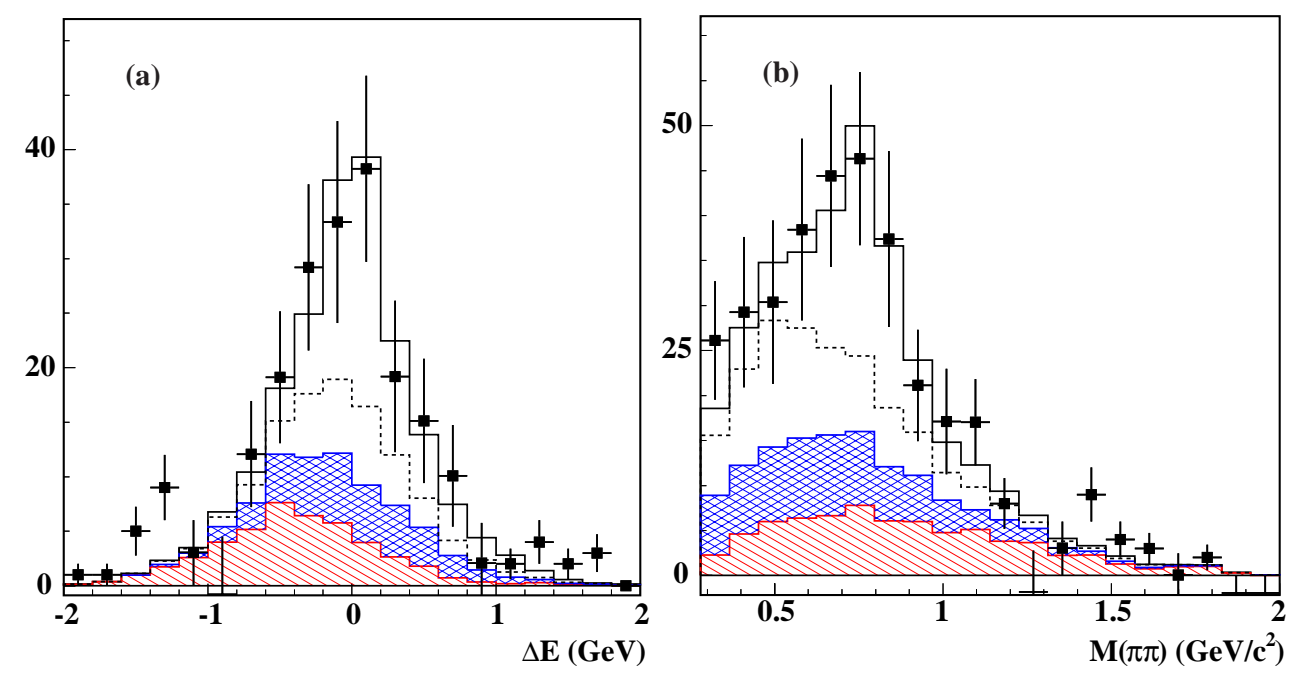

Figure 7: Projections of maximum likelihood fit for HILEP energy bin: (a) $\Delta E$ distribution with the cut $\left|M(\pi \pi)-M_{\rho}\right|<0.15 \mathrm{GeV} / c^{2}$ and (b) $M(\pi \pi)$ distribution with the cut $\Delta E<500 \mathrm{MeV}$. The points are the continuum subtracted data. The solid histogram is the fit, represented as the sum of three components: signal and cross-feed (open regions), background from non-signal $b \rightarrow u \ell \nu$ (double-hatched region) and background from $b \rightarrow c \ell \nu$ (single-hatched region).

$b \rightarrow u \ell \nu$ (modes other than $\rho, \omega$ and $\pi$ ) and $b \rightarrow c \ell \nu$. The fit also contains background contributions from continuum and fake leptons; we measure these contributions using off-resonance data and known fake rates. The signal shapes for the fit are taken from Monte Carlo simulation using various form factor models for $B \rightarrow \rho \ell \nu$ and

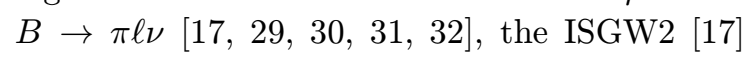
model for $b \rightarrow u \ell \nu$ and a combination of ISGW2 and CLEO form factor results [1] 긴, for $b \rightarrow c \ell \nu$. Isospin and quark model relations are used to constrain the relative normalizations of the three vector modes $\left(B^{0} \rightarrow \rho^{-} \ell^{+} \nu, B^{+} \rightarrow \rho^{0} \ell^{+} \nu\right.$ and $\left.B^{+} \rightarrow \omega \ell^{+} \nu\right)$ and, separately, the normalizations of the pseudoscalar modes $\left(B^{0} \rightarrow \pi^{-} \ell^{+} \nu\right.$ and $\left.B^{+} \rightarrow \pi^{0} \ell^{+} \nu\right)$. Our fit also accounts for the large cross-feed between the various signal modes.

We find statistically significant yields for $B \rightarrow$ $\rho \ell \nu$. Figure $\bar{i}$ shows projections of the maximum likelihood fit for $\pi^{+} \pi^{-}$and $\pi^{ \pm} \pi^{0}$ modes in the high energy bin onto the variables $\Delta E$ and $M(\pi \pi)$ overlayed with the data. We average over the various form factor models for $\rho \ell \nu$ and $\pi \ell \nu$, finding

$$
\begin{aligned}
\mathcal{B}(B \rightarrow \rho \ell \nu) & =\left(2.69 \pm 0.41_{-0.40}^{+0.35} \pm 0.50\right) \times 10^{-4} \\
\left|V_{u b}\right| & =\left(3.23 \pm 0.24_{-0.26}^{+0.23} \pm 0.58\right) \times 10^{-3}
\end{aligned}
$$

where the errors are statistical, systematic and due to model dependence. These results for $\mathcal{B}(B \rightarrow$ $\rho \ell \nu)$ and $\left|V_{u b}\right|$ are consistent with the neutrino reconstruction analysis [24]. The two results are statistically independent, but the systematic and model dependence uncertainties are largely correlated. Taking into account the correlations, the combined results are

$$
\begin{aligned}
\mathcal{B}\left(B^{0} \rightarrow \rho^{-} \ell^{+} \nu\right) & =\left(2.57 \pm 0.29_{-0.46}^{+0.33} \pm 0.41\right) \times 10^{-4} \\
\left|V_{u b}\right| & =\left(3.25 \pm 0.14_{-0.29}^{+0.21} \pm 0.55\right) \times 10^{-3}
\end{aligned}
$$

The $\pi \ell \nu$ mode is dominated by cross-feed from $\rho \ell \nu$, but the branching fraction $\mathcal{B}(B \rightarrow \pi \ell \nu)=$ $(1.3 \pm 0.4) \times 10^{-4}$ (statistical error only) is consistent with the neutrino reconstruction analysis. In $\omega \ell \nu$, the fit describes the data well but we do not observe a significant signal.

We are also able to measure the $q^{2}$ distribution for $B \rightarrow \rho \ell \nu$ events with $E_{\ell}>2.3 \mathrm{GeV}$. Figure $\overline{\beta_{1}^{\prime}}$ shows the data distribution of $q^{2}$ after requiring $\left|M(\pi \pi)-M_{\rho}\right|<0.15 \mathrm{GeV} / c^{2}$ and $\Delta E<500 \mathrm{MeV}$. We quote partial widths for three $q^{2}$ bins in table 2 . The measurements are subject to statistical, systematic and model dependence uncertainties. The model dependence uncertainty comes primarily from the extrapolation to all lepton energies. In the highest $q^{2}$ 

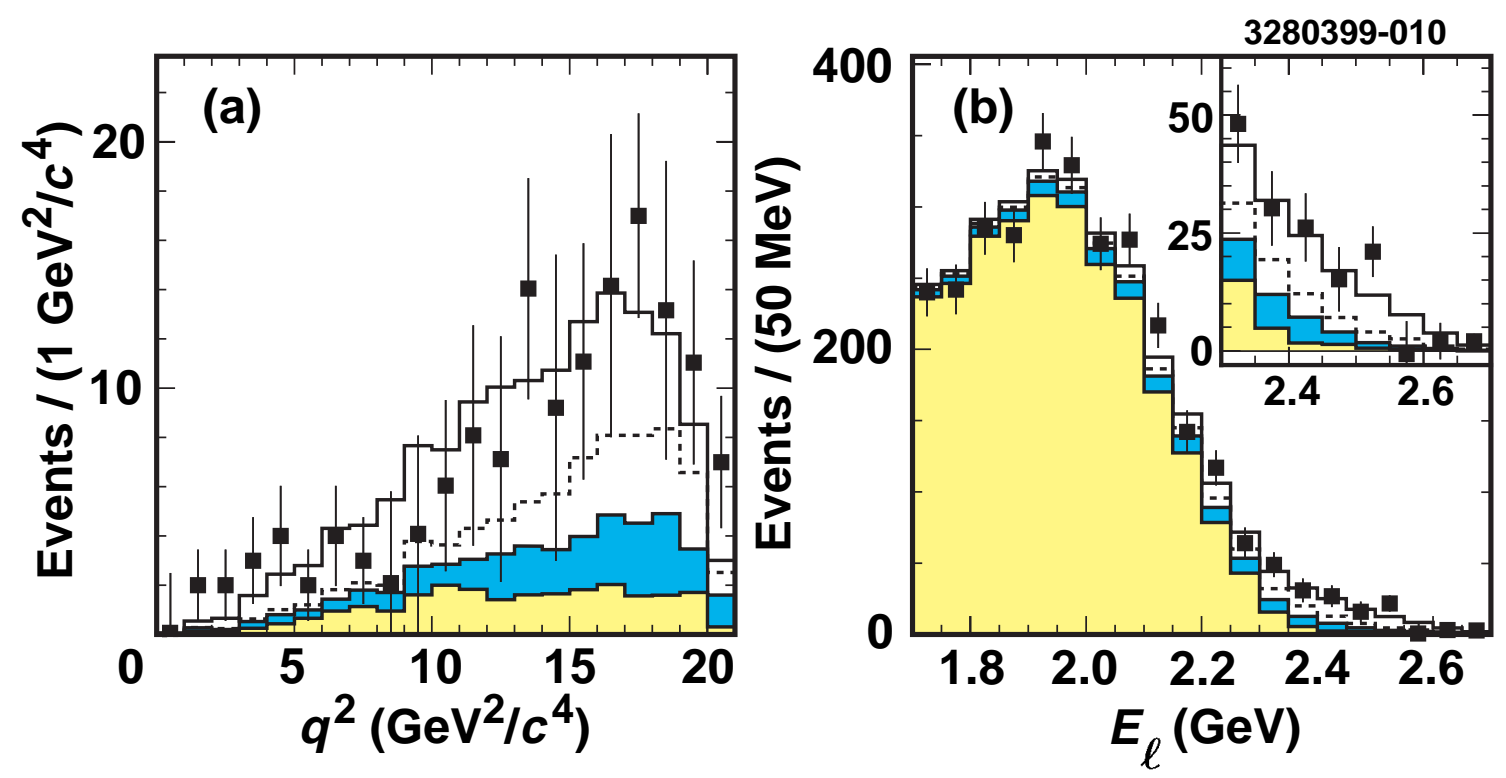

Figure 8: The projections of the fit onto $q^{2}$ (a) and $E_{\ell}$ (b) for HILEP after the cuts $\Delta E<500 \mathrm{MeV}$ and $\left|M(\pi \pi)-M_{\rho}\right|<0.15 \mathrm{GeV} / c^{2}$. The points show the on-resonance data after continuum subtraction, while the histogram shows the projection of the fit. The contributions to the fit are direct and cross-feed components of the signal (unshaded regions above and below the dashed line), the background from $b \rightarrow u \ell \nu$ non-signal modes (darkly shaded region) and background from $b \rightarrow c \ell \nu$ (lightly shaded region).

$$
\begin{aligned}
\Delta \Gamma\left(0<q^{2}<7 \mathrm{GeV}^{2} / c^{4}\right) & =\left(7.6 \pm 3.0_{-1.2}^{+0.9} \pm 3.0\right) \times 10^{-2} \mathrm{~ns}^{-1} \\
\Delta \Gamma\left(7<q^{2}<14 \mathrm{GeV}^{2} / c^{4}\right) & =\left(4.8 \pm 2.9_{-0.8}^{+0.7} \pm 0.7\right) \times 10^{-2} \mathrm{~ns}^{-1} \\
\Delta \Gamma\left(14<q^{2}<21 \mathrm{GeV}^{2} / c^{4}\right) & =\left(7.1 \pm 2.1_{-1.1}^{+0.9} \pm 0.6\right) \times 10^{-2} \mathrm{~ns}^{-1}
\end{aligned}
$$

Table 2: The partial width $\Delta \Gamma$ for $B \rightarrow \rho \ell \nu$ in bins of $q^{2}$.

bin, the model dependence is small because the experimentally accessible lepton energies $\left(E_{\ell}>\right.$ $2.3 \mathrm{GeV}$ ) cover fractionally more of the allowed phase space. We compare the measured differential decay rate to expectations from different form factor models in figure $\overline{\bar{p}_{\mathbf{r}}^{\prime}}$ At high lepton energy, the form factor models agree well on the shape of the form factor, which is dominated by one of three relevant form factor terms $\left(A_{1}\left(q^{2}\right)\right)$, and in fact the model dependence might be quite small. In a future analysis, one might choose to measure $\left|V_{u b}\right|$ using the decay rate at large $q^{2}$. At the same time, the good agreement removes the possibility of differentiating between models at this time. Measurement of the partial rate for $E_{\ell}<2.0 \mathrm{GeV}$ would help improve the form factor models, and thus improve measurement of $\left|V_{u b}\right|$. It is also possible lattice QCD calculations can provide more precise information about the form factor in an experimentally accessible region of $q^{2}$ and $E_{\ell}$.

\section{Analysis of Inclusive $B \rightarrow X_{c} \ell \nu$}

Inclusive measurements of $b \rightarrow c \ell \nu$ also give information on $\left|V_{c b}\right|$. CLEO has two preliminary results based on inclusive techniques for measuring semileptonic decays [333, 34]. Both rely on Heavy Quark Effective Theory (HQET) and the operator product expansion (OPE) to interpret the results. Within this framework an inclusive measurement summed over many final states is readily interpreted from quark level calculations.

The rate for inclusive $B \rightarrow X_{c} \ell \nu$ is proportional to $\left|V_{c b}\right|^{2}$. In the OPE and HQET to order 


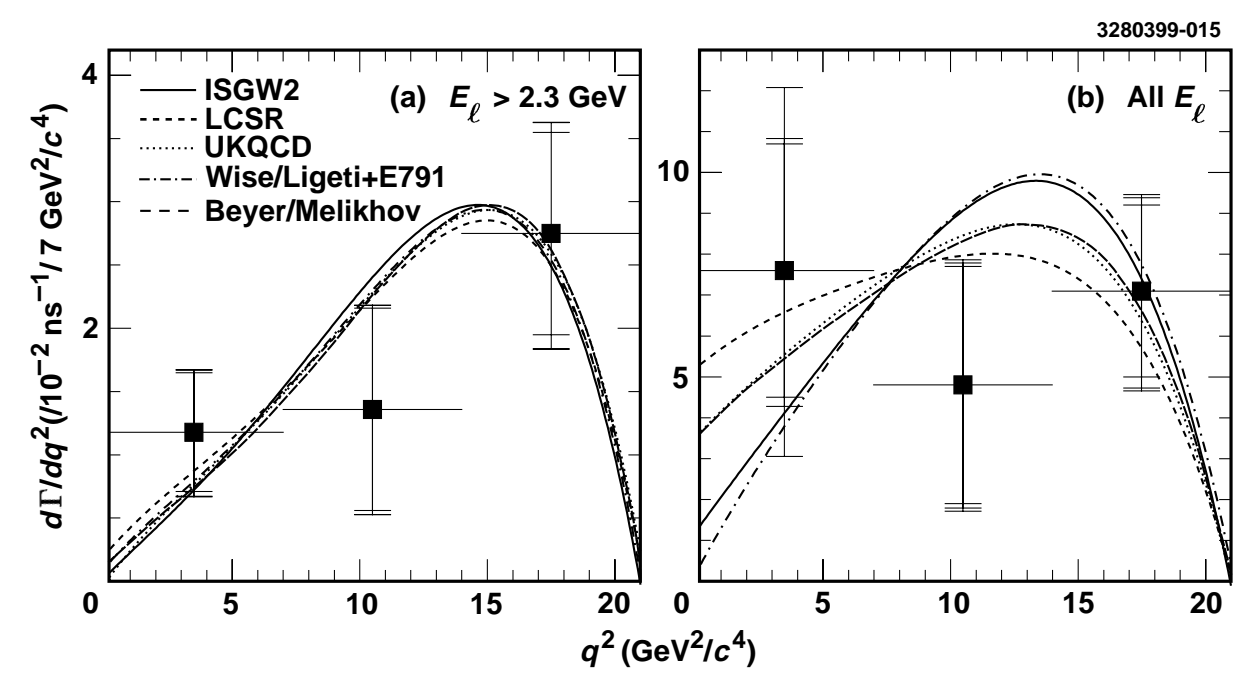

Figure 9: Comparison of measured differential rate $d \Gamma / d q^{2}$ in three bins and expectations from five form factor models. (a) shows the results for $E_{\ell}>2.3 \mathrm{GeV} / c$, while (b) extrapolates the data to the entire lepton energy range.

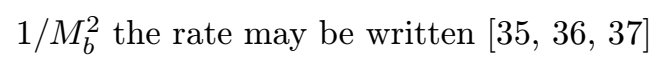

$$
\begin{aligned}
\Gamma_{s l}= & \frac{G_{F}^{2}\left|V_{c b}\right|^{2} M_{B}^{5}}{192 \pi^{3}} 0.369\left[1-1.54 \frac{\alpha_{s}}{\pi}\right. \\
& -1.65 \frac{\bar{\Lambda}}{M_{B}}\left(1-0.87 \frac{\alpha_{s}}{\pi}\right)-0.95 \frac{\bar{\Lambda}^{2}}{M_{B}^{2}} \\
& \left.-3.18 \frac{\lambda_{1}}{M_{B}^{2}}+0.02 \frac{\lambda_{2}}{M_{B}^{2}}\right]
\end{aligned}
$$

The parameters $\lambda_{1}$ and $\lambda_{2}$ are matrix elements of the HQET expansion, which have the following intuitive interpretations: $\lambda_{1}$ is proportional to the kinetic energy of the $b$-quark in the $B$ meson and $\lambda_{2}$ is the energy of the hyperfine interaction of the $b$-quark spin and the light degrees of freedom in the meson. $\bar{\Lambda}$ is introduced to relate the $b$-quark and $B$ meson masses, representing the energy of the light degrees of freedom.

From the $B-B^{*}$ mass difference, $\lambda_{2}$ is determined to be $0.12 \mathrm{GeV}^{2} . \bar{\Lambda}$ and $\lambda_{1}$ are more difficult to determine, but if they can be measured, one can measure $\left|V_{c b}\right|$ given $\Gamma_{s l}$. For example,

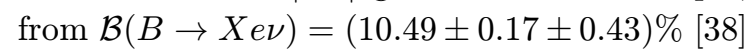
and the average $B$ lifetime $\tau_{B}=1.61 \pm 0.02 \mathrm{ps}$ one finds $\Gamma_{s l}=65.0 \pm 3.0 \mathrm{~ns}^{-1}$. At present our knowledge of $\lambda_{1}$ and $\bar{\Lambda}$ limits the precision we can achieve on $\left|V_{c b}\right|$ from inclusive semileptonic $B$ decays. The aim of the new inclusive analyses is to determine $\lambda_{1}$ and $\bar{\Lambda}$ from experiment and thereby decrease the theoretical uncertainty which comes when extracting $\left|V_{c b}\right|$ from $\Gamma_{s l}$. Each analysis alone provides two constraints, allowing a measurement of $\bar{\Lambda}$ and $\lambda_{1}$. Combining the two analyses over-constrains the theory parameters thus allowing a test of the theoretical framework and experimental understanding of $b$-quark decays.

\subsection{Hadronic Mass Moments}

For decays $B \rightarrow X_{c} \ell \nu$, the first method measures the first and second hadronic mass moments. Falk et al. [355] give an expansion for the moments of the squared hadronic invariant mass $\left(M_{X_{c}}^{2}\right)$ distribution in the variables $1 / M_{B}$ and $\alpha_{s}$. The moments have been calculated integrated over all lepton energies $\left[3 \overline{3} \overline{5}_{1}^{1}, \overline{1}_{2} \overline{7}_{1}\right]$ and subject to a necessary experimental cut on the lepton energy [36]. Equations 5.21 and 5.3 give the expansions for the first and second moments to order $1 / M_{B}^{2}$, for $E_{\ell}>1.5 \mathrm{GeV}$.

$$
\begin{aligned}
\left\langle M_{X_{c}}^{2}\right. & \left.-\bar{M}_{D}^{2}\right\rangle=M_{B}^{2}\left[0.0272 \frac{\alpha_{s}}{\pi}\right. \\
& +0.207 \frac{\bar{\Lambda}}{M_{B}}\left(1+0.43 \frac{\alpha_{s}}{\pi}\right) \\
& \left.+0.193 \frac{\bar{\Lambda}^{2}}{M_{B}^{2}}+1.38 \frac{\lambda_{1}}{M_{B}^{2}}+0.203 \frac{\lambda_{2}}{M_{B}^{2}}\right] \\
\left\langle\left(M_{X}^{2}-\bar{M}_{D}^{2}\right)^{2}\right\rangle & =M_{B}^{4}\left[0.00148 \frac{\alpha_{s}}{\pi}+\right.
\end{aligned}
$$



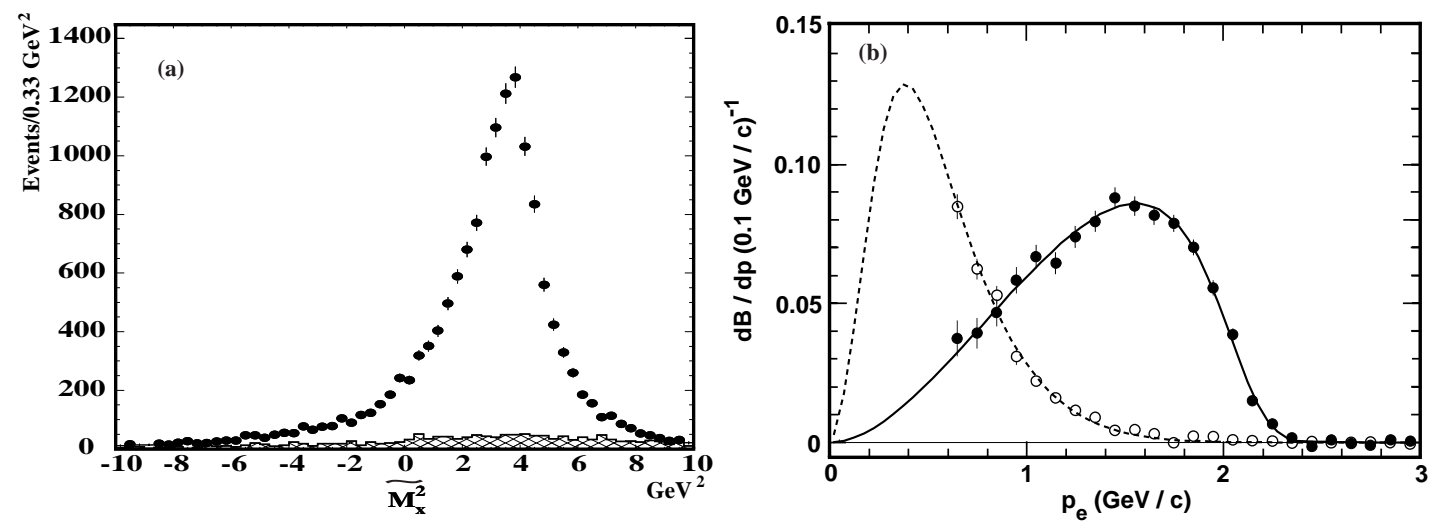

Figure 10: (a) Measured $\tilde{M}_{X_{c}}^{2}$ distributions for on-resonance data (points) and scaled off-resonance data (hatched histogram). (b) Electron momentum spectrum from $B \rightarrow X e \nu$ (solid circles) and $b \rightarrow c \rightarrow Y e \nu$ (open circles). The curves show the best fit to the ISGW model with $23 \% B \rightarrow D^{* *} \ell \nu$.

$$
\left.+0.038 \frac{\bar{\Lambda}}{M_{B}} \frac{\alpha_{s}}{\pi}+0.0535 \frac{\bar{\Lambda}^{2}}{M_{B}^{2}}-0.12 \frac{\lambda_{1}}{M_{B}^{2}}\right]
$$

The moments are defined relative to the spinaveraged $D$ meson mass, $\bar{M}_{D}=1.975 \mathrm{GeV} / c^{2}$. By measuring the first two moments and inverting the equations one may determine or constrain the remaining HQET parameters $\lambda_{1}$ and $\bar{\Lambda}$.

To measure the hadronic mass moments in semileptonic $B$ decays we select events with one lepton of momentum $p_{\ell}>1.5 \mathrm{GeV} / c$. We "reconstruct" the neutrino using the hermeticity of the detector, imposing strict event quality cuts to ensure no particles are missed. The net charge of the event must be zero, and the missing mass must be consistent with a neutrino. The mass recoiling against the lepton and neutrino is:

$$
\begin{aligned}
M_{X_{c}}^{2}= & M_{B}^{2}+M_{\ell \nu}^{2}-2 E_{B} E_{\ell \nu} \\
& +2\left|\mathbf{p}_{B}\right|\left|\mathbf{p}_{\ell \nu}\right| \cos \theta_{\ell \nu-B} .
\end{aligned}
$$

Since the $B$ momentum is small but the direction is unknown, we approximate $M_{X_{c}}^{2}$ by dropping the last term.

$$
\widetilde{M_{X_{c}}^{2}}=M_{B}^{2}+M_{\ell \nu}^{2}-2 E_{B} E_{\ell \nu}
$$

The resulting distribution shown in figure 10 ia has contributions from $b \rightarrow c \ell \nu$ (96\%), $b \rightarrow c \rightarrow$ $s \ell \nu(3 \%)$ and $b \rightarrow u \ell \nu(1 \%)$. We compute the moments after background subtraction using MC shapes. We further correct for a bias in the reconstructed hadronic mass due to asymmetric resolution of the neutrino reconstruction. We find

$$
\left\langle M_{X_{c}}^{2}-\bar{M}_{D}^{2}\right\rangle=0.286 \pm 0.023 \pm 0.080 \mathrm{GeV}^{2}
$$

$$
\left\langle\left(M_{X_{c}}^{2}-\bar{M}_{D}^{2}\right)^{2}\right\rangle=0.911 \pm 0.066 \pm 0.309 \mathrm{GeV}^{4} .
$$

\subsection{Lepton Energy Moments}

The second method uses the inclusive electron spectrum from $B$ decays measured by CLEO Theoretical expressions for the moments of the lepton spectrum are given by Voloshin [39 $\left.\overline{3}^{\prime}\right]$. As in the case of the hadronic mass moments, these expressions may be inverted to place constraints on $\lambda_{1}$ and $\bar{\Lambda}$.

The $B \rightarrow X e \nu$ electron spectrum measure-

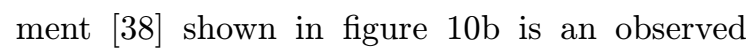
spectrum above $0.6 \mathrm{GeV}$. In events with a high momentum lepton tag and an additional electron, the primary electrons $\left(b \rightarrow c \ell^{-} X\right)$ are separated from secondary electrons from charm decays $\left(b \rightarrow c X ; c \rightarrow \ell^{+} Y\right)$ using angular and charge correlations. To measure the moments and compare to theory, we must apply corrections to the observed primary spectrum. We extrapolate below $0.6 \mathrm{GeV}$ and correct for detector smearing (including bremsstrahlung) and motion of the $B$ in the lab frame. There are also electromagnetic radiative corrections which are not included in the theoretical expressions for the moments. After all corrections we find the following preliminary results.

$$
\begin{aligned}
\left\langle E_{\ell}\right\rangle & =1.36 \pm 0.01 \pm 0.02 \mathrm{GeV} \\
\left\langle\left(E_{\ell}-\left\langle E_{\ell}\right\rangle\right)^{2}\right\rangle & =0.19 \pm 0.004 \pm 0.005 \mathrm{GeV}^{2}
\end{aligned}
$$

\subsection{Interpretation of Moments}

Inverting the theoretical expression for the hadronic 


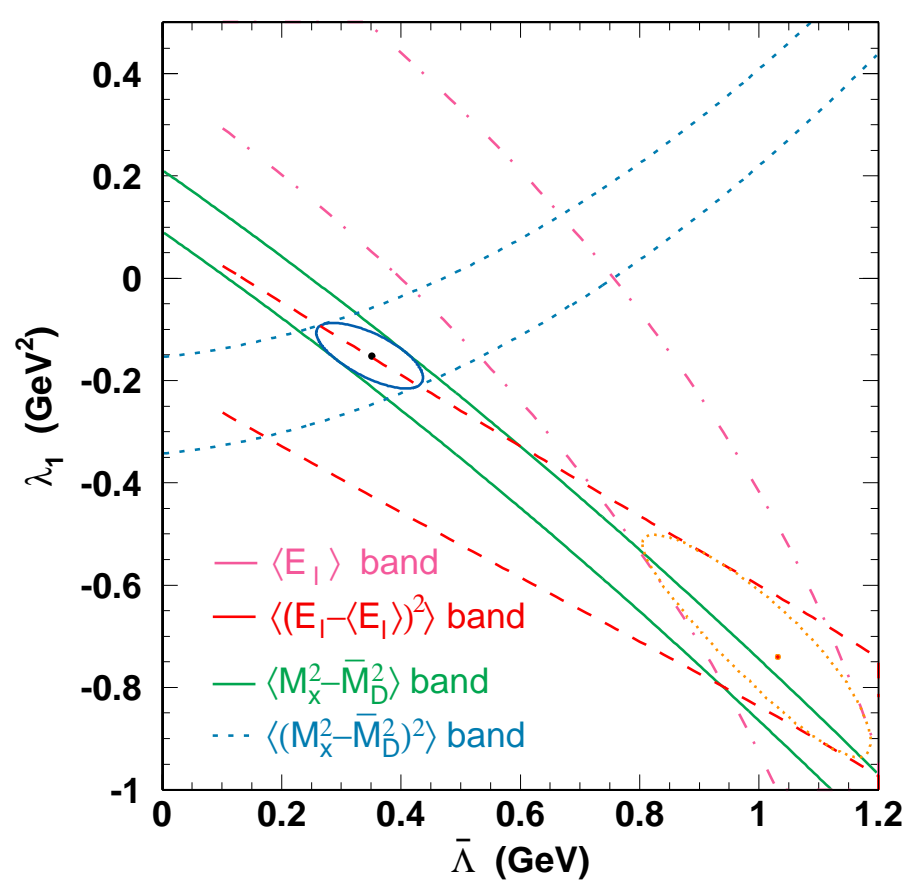

Figure 11: Combined constraints on HQET parameters $\lambda_{1}$ and $\bar{\Lambda}$ from hadronic recoil mass moments and lepton energy spectrum moments.

moments to $\mathcal{O}\left(1 / M_{B}^{2}\right)$ and solving for HQET parameters gives the results

$$
\begin{aligned}
\bar{\Lambda} & =+0.33 \pm 0.02 \pm 0.08 \mathrm{GeV} \\
\lambda_{1} & =-0.13 \pm 0.01 \pm 0.06(\mathrm{GeV} / c)^{2} .
\end{aligned}
$$

Equivalently, each moment measurement provides a constraint in the $\lambda_{1}-\bar{\Lambda}$ plane. The allowed bands and overlap region are shown in figure $\overline{1}_{\mathbf{L}} \overline{1}_{-}^{1}$. The $1 \sigma$ allowed regions shown in the figure include statistical and both experimental and theoretical systematic uncertainties. We use the $\mathcal{O}\left(1 / M_{B}^{3}\right)$ expansion of the moments $\left[3 \overline{6}^{4}\right]$ to estimate the effect of higher order terms in the hadronic moments calculation. Variations of the $1 \sigma$ contours shown include this theoretical systematic uncertainty.

The lepton moment measurements can also be converted to allowed bands in the $\lambda_{1}-\bar{\Lambda}$ plane (figure $\left.{ }_{1}^{1} \overline{1}_{1}^{1}\right)$. In these preliminary analyses, the agreement among the four allowed bands is only at the $5-10 \%$ confidence level. Taken at face value, the hadron moment measurement alone implies a $3 \%$ measurement of $\left|V_{c b}\right|$. However, if one uses the central value from the lepton moments instead, $\left|V_{c b}\right|$ shifts by $\sim 10 \%$. Clearly before we can feel comfortable with precision de- terminations of $\left|V_{c b}\right|$ or $\left|V_{u b}\right|$ from inclusive measurements, we must resolve the discrepancy.

A few comments on the current discrepancy are in order. First, Falk et al. expect the second order hadronic mass moments to be more sensitive to higher order corrections and therefore less reliable than the first moment [3 $3 \overline{5}]$. However, an attempt has been made to include the theoretical uncertainty in the systematic errors as described above. The theoretical uncertainty from the lepton energy moments is harder to estimate, because these moments are presently calculated only to second order in $1 / M_{B}$. A resolution of the discrepancy may require higher order expansions for the lepton energy moments.

Second, something may be wrong with the measurement of the lepton energy moments. Ligeti has questioned the lepton moment measurement since it has some model dependence from the extrapolation below $0.6 \mathrm{GeV}\left[40_{1}^{\prime}\right]$. Another potential problem: there may be additional sources of leptons in the CLEO data other than those considered in [38]. Primary and secondary leptons are separated using charge and kinematic correlations after removing leptons from $\psi^{\left({ }^{\prime}\right)}, D_{s}$ and $\Lambda_{c}$ decays. Besides $\bar{D}$ 's from the lower-vertex 
in $B$ decays $\left(\bar{b} \rightarrow \bar{c} W^{+}\right)$, there can also be $D$ 's from the upper-vertex $\left(W^{+} \rightarrow c \bar{s}\right)$. The contam-

- ination of the_observable lepton spectrum_from decays of such upper-vertex $D$ 's should be small because of the relatively small branching fraction and the lower available energy given the presence of two $D$ mesons in the decay. However, in light of recent results for upper-vertex $D$ production in $B$ decays $\left[\tilde{H}_{1} \overline{1}_{1}\right.$, the contribution from this source of background needs to be revisited.

Finally, it has been noted that moments of the photon energy spectrum in $b \rightarrow s \gamma$ could provide constraints on $\bar{\Lambda}$ and $\lambda_{1}\left[\overline{4} \overline{2}_{2}\right]$. For example, - thre-width - of the photorr spectrumn-(mreasured in

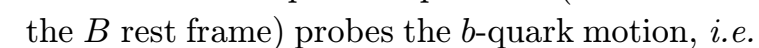
- $\lambda_{\text {r.- But, }}$ it-is -important te-note that the back- grounds in $-b \rightarrow s \gamma$-are-very large, requiring an experimental cut on the photon energy [4]

If the discrepancy between the two moment techniques remains after further analysis we may have to question the assumption of quark-hadron duality implicit in such inclusive analyses.

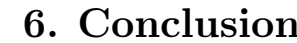

The CLEO measurements of the $B \rightarrow D^{(*)} \ell \nu$ - form- facters-antct $q^{2}$ - distribution $\dot{\text { int }}-B \rightarrow-\rho \ell v-$ - show progress in the experimentalumderstanding- of-the-dynamics of beavy-quark-decay - This -un- elerstanding,-cøupled -nxith mюre theoretical wørk,

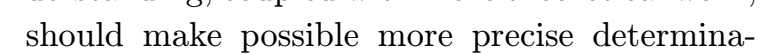
L tions $\mid V_{c b} b$ and $\left|V_{u b}\right|-$ - Jikewise moment_ based_. analyses of inclusive semileptonic $B$ decays seek to use data to constrain theory parameters and thereby reduce the uncertainties in extracting $\left|V_{c b}\right|$ from the inclusive rate for $b \rightarrow \bar{c} l \nu$.

Rèfērèn̄n̄ēēs i - [1] N. Cabibbo, , Ph h '- - Phys. 49

- - 334 _Y._Kubata et al, _Nuch Jnstr. _Meth_ A_320, 66 :-

[4] M. E. Luke, Physs. Lett. B 252 (1990) 447.

[5l M. Neubert, 'Phys. Lett. B 264 (1991) 455 ;

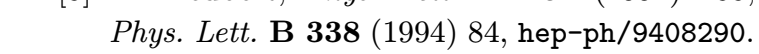

[6]_A. Falk and M. Neubert, Phys. Rev. D 47 [ - _ _ (1993)_2965, hep-ph/9209268'
[7] M. Shifman et al., IPhys. Rev. D 51 (1995) 2217, hep-ph/9405207i ?Phys. Rev. D _ 52! (1995) 3149.

[8] A. Czarnecki, Phys. Rev. Lett. 76 (1996) 4124, hep-ph/9603261.

[9] P.F. Harrison and H.R. Quinn, Editors, The BaBar Physics Book, SLAC-R-504, 1998.

[10] I. I. Bigi, 'hep-ph/9907270;

[11] B. Barish et al., 'Phys. Rev.

[12] C. Caso et al., Eur. Phys. J.'C $\mathbf{3}(1999) 1$.

[13] M. Calvi, these proceedings.

[14] M. Athanas et al., 'Phys. Rev. Lett. 799 (1997)i 2208

[15] J. Bartelt et al., ,'Phys. Rev. Lett. 82 3746

[16] N. Isgur et al., 'Phys. Rev. D_39 (1989) 7999,

[17] D. Scora and N. Isgur, 'Phys. Rev. D 52 (1995), 2783, hep-ph/9503486,

[18] J. E. Duboscq et al., 'Phys. Rev.Lett. $\mathbf{7 9}$ (1996)! 3898

[19] J. Goity and W. Roberts, Phys. Rev. D 51 $(1995) 3459$, hep-ph/9406236!

[20] C. G. Boyd, _ B. _ Grinstein, _ and R. F. Lebed, Phys. Rev. D $56 .(1997)$ 6895, hep-ph/9705252.

[21] I. Caprini and M. Neubert, 'Phys. Lett. B-380 (1996) 376, hep-ph/9603414

[22]_ I. Caprini, L. Lellouch and M. Neubert, 'Nucl.' Phys. B 530 (1998) 153, hep-ph/9712417'

[23] S. Hashimoto et al., ,Phys. Rev. D 61 (1999)' 014502, hep-ph/9906376.

[24] J. Alexander et al., 'Phys. Rev. Lett. 77_(1996), $5000 !$

[25] M. Wirbel, B. Stech and M. Bauer, 'Z. Physih C $29(1985) 637$

[26] J. G. Kö̈ner and G. A. Schuler, 'Z. Physik $38(1988) 511$

[27] D. Melikhov, 'Phys. Rev. D $\mathbf{5 3}(1996) 246 \overline{0}$ hep-ph/9509268: Phys. Lett. B 380 (1996)! 363, hep-ph/9603340,

[28] B. H. Behrens et al., hep-ex/9905056̈.

[29] P. Ball and V. M. Braun, Phys. Rev. D 58! $(1998) 094016_{n}^{\prime}$, hep-ph/9805422

[30] M. Beyer and D. Melikhov, 'Phys. Lett. B.436' (1998) 344, hep-ph/9807223. 
[31]_ L. Del. Debbio et al., 'Physs. Lett.B.416.(1998)' -

[32] Z. Ligeti and M. B. Wise, 'Phys. Rev.D 53' ; (1996) 4937̈tand E. M. Aitala et al., 'Phys. Rev.' .

[33] J. Bartelt et al., ICHEP98 1013, CLEO CONF 98-21 (1998).

[34] S. E. Roberts, Ph.D. thesis, Univ. of Rochester (1997).

[35] A. Falk, M. Luke, M. Savage, Phys. Rev. D 53 (1996) 2491' hep-ph/9507284 and Phys. Revi! _ _ _ D 53 (1996) 6316! hep-ph/9511454:

[36] A. Falk and M. Luke, Phys. Rev. D 57 (1998) ( hep-ph/9708327.

[37] M. Gremm and A. Kapustin, 'Phys. Rev. D.55! ; - _ (1997) 6924, hep-ph/9603448;

[38] B. Barish et al., 'Physs. Rev. Lett. 76 $\mathbf{6}$ (1995)', - - - 1570

[39] M. Voloshin, 'Phys. Rev. D 51 hep-ph/9411296i

[40] Z. _ Ligeti, - hep-ph/9904460 1 and hep-ph/9908432i

[41] T. E. Coan et al., 'Phys. Rev. Lett. 80 (1998)'

: - _ 1150' hep-ex/9710028:

[42] C. Bauer, IPhys. Rev. D. 57. (1998) 5611, hep-ph/9710513'

[43] D. Jaffe, these proceedings, hep-ex/9910055. 